\title{
BACKGROUND RECOGNITION USING NEURAL NETWORK METHODS*
}

\author{
Sebastian Kupny, Janusz BrzychCZyk \\ The Marian Smoluchowski Institute of Physics, Jagiellonian University \\ Reymonta 4, 30-059 Kraków, Poland \\ Jerzy Łukasik, Piotr PaWŁowski \\ The Henryk Niewodniczański Institute of Nuclear Physics PAN \\ Radzikowskiego 152, 31-342 Kraków, Poland
}

(Received July 29, 2013)

The Kohonen Self Organizing Neural Network has been used to recognize the background from secondary reactions in long $\mathrm{CsI}(\mathrm{Tl})$ crystals. It has been applied to the experimental data from the ASY-EOS campaign conducted at GSI, and allowed to reduce the background by $75 \%$.

DOI:10.5506/APhysPolBSupp.6.1115

PACS numbers: 21.65.Mn, 21.65.Cd, 29.40.Wk, 07.05.Mh

Heavy ion collisions at high energies offer a unique opportunity to study the properties of nuclear matter at extreme conditions. The reaction products from such collisions are usually measured using the $\Delta E-E$, time-offlight or magnetic spectrometer methods (see e.g. [1]). One of the limitations of the standard $\Delta E-E$ telescope method are the secondary reactions in the detector material induced by energetic primary products. The probability of such reactions increases with the range, and thus with the energy, of the measured product, and limits the application of the method to reactions with energies up to several hundreds of $\mathrm{MeV} /$ nucleon. For the ${ }^{197} \mathrm{Au}+{ }^{197} \mathrm{Au}$ collisions at $400 \mathrm{MeV} /$ nucleon studied in this presentation, the probabilities of secondary reactions reach up to $30-40 \%$ for energetic neutrons and light charged particles stopped in the $15 \mathrm{~cm}$ long $\mathrm{CsI}(\mathrm{Tl})$ active layer of the KRATTA modules [2].

* Presented at the Symposium on Applied Nuclear Physics and Innovative Technologies, Kraków, Poland, June 3-6, 2013. 
The KRATTA array has been built to detect light charged reaction products in the ASY-EOS experiment [3]. The experiment has been designed to study the density dependence of the nuclear symmetry energy by measuring flows and isotopic compositions of the reaction products from the ${ }^{197} \mathrm{Au}+{ }^{197} \mathrm{Au},{ }^{96} \mathrm{Ru}+{ }^{96} \mathrm{Ru}$, and ${ }^{96} \mathrm{Zr}+{ }^{96} \mathrm{Zr}$ reactions at $400 \mathrm{MeV} /$ nucleon. It has been conducted at GSI Darmstadt in 2011. The KRATTA array consists of 38 independent modules. Each module is a triple $\Delta E-\Delta E-E$ telescope composed of two $\mathrm{CsI}(\mathrm{Tl})$ scintillation crystals of 2.5 and $12.5 \mathrm{~cm}$ length, each coupled to a $500 \mu \mathrm{m}$ thick, large area PIN photodiode, used for both light readout and direct (ionization) detection. An additional photodiode has been placed in front of the thin crystal and served as a first $\Delta E$ layer for direct detection only. The signals from photodiodes were integrated by charge preamplifiers and digitized with $100 \mathrm{MHz}, 14$ bits Flash ADCs. The stored waveforms spanned $10.24 \mu \mathrm{s}$ (1024 time bins), with a $2 \mu$ s pre-trigger enabling a precise baseline estimation.

The off-line pulse shape analysis [4] allowed to decompose the complex photodiode signals into three components: the direct (ionization) signal and the fast and slow light components from the scintillators. These three components differ by their decay times and their individual strengths. The method of deconvolving the signals consisted in using the parametrized shapes of the three components, finding the baseline and the starting time of the signal, and using the minimization algorithm to determine the amplitudes and the rise and fall times. The obtained amplitudes were used to construct $\Delta E-E$ identification maps. Figure 1 (a) shows a $\Delta E-E$ map for scintillation signals from the CsI crystals. The observed lines correspond to $p, d, t,{ }^{3} \mathrm{He}, \alpha$, and so on, from bottom to top. Apart from the
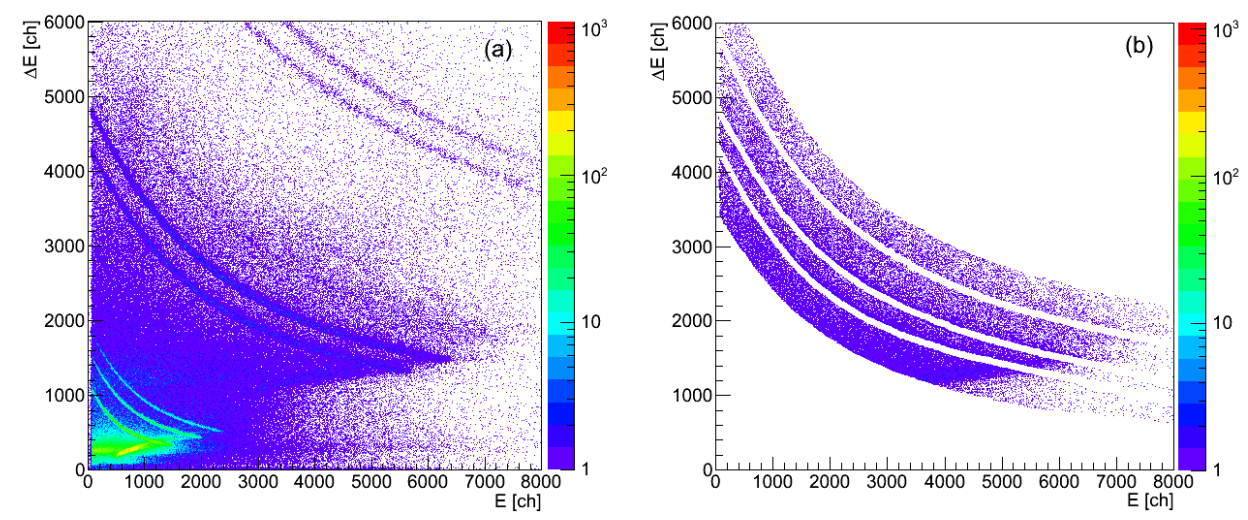

Fig. 1. The $\Delta E-E$ maps: (a) all the data used in the analysis, (b) the training set for the KNN. 
very good isotopic resolution, one can see a substantial background from the secondary reactions/scatterings in the crystals and also from particles crossing the module at an angle and not originating from the target, as well as back-bendings corresponding to particles punching through the thick crystal.

The particle identification is done by setting the graphical cuts on the lines in the $\Delta E-E$ maps. However, the hits identified in this way contain a significant background contribution. In this work, the pulse shape parameter space analysis has been performed, with the application of neural networks, in order to recognize the background hits also underneath the isotopic lines. At the first stage, the analysis was focused on the He isotopes. The training set for neural networks has been defined by the background hits located in the vicinity of the ${ }^{3} \mathrm{He},{ }^{4} \mathrm{He}$ and ${ }^{6} \mathrm{He}$ isotope lines, as depicted in Fig. 1 (b).

The background recognition has been performed using the Kohonen Neural Network (KNN) [5], which is a type of self-organizing neural networks. KNN consists of one layer of neurons and uses a method of unsupervised competitive learning. The network was created from one-dimensional lattice of 1000 neurons. Each neuron is associated with a vector of weights of the same dimension as the input vector. The input vector contains six elements that characterize the fast and slow scintillation components from both CsI crystals. They are the fast/slow amplitude ratios and the rise times. To apply KNN, the first step is training to determine the appropriate weight set. Prior to training, the weights of neurons are initialized by random values. After reading an input vector from the training data set, the neuron whose weight vector is most similar to the input is identified. It is called the best matching unit (BMU). The weights of the BMU and neighbour neurons are then adjusted towards the input vector. The weight modification procedure is repeated for all the hits of the training set.

After the teaching process, the trained KNN was used to select a subset of neurons sensitive to events lying on isotopic lines or those constituting the background. Each examined event (input vector) was assigned to the number of its BMU, used as a selecting variable. The selection of backgroundsensitive neurons was based on calculating the Signal-to-Noise Ratio (SNR). The real atomic mass was assigned to each hit during the identification procedure and reflected a distance of the hit position from the ridge of the nearest isotopic line. The SNR factor was defined as a ratio of the number of events above/below the smooth background fit to the real mass distribution using the TSpectrum class of ROOT [6]. The SNR cut condition was set to $25 \%$.

After applying the described neuron selection method, the neuron distribution was split into two parts: one consisted of neurons sensitive to the background events and the other - sensitive to hits close to the He isotopic 
lines. Figure 2 (b) shows the identification map for the first, and Fig. 2 (a) for the second class of neurons. As can be seen, the method allowed to split the original identification map of Fig. 1 (a) into a smooth background distribution in the region of He isotopes including the punch-through segments and a distribution with pronounced isotopic lines.
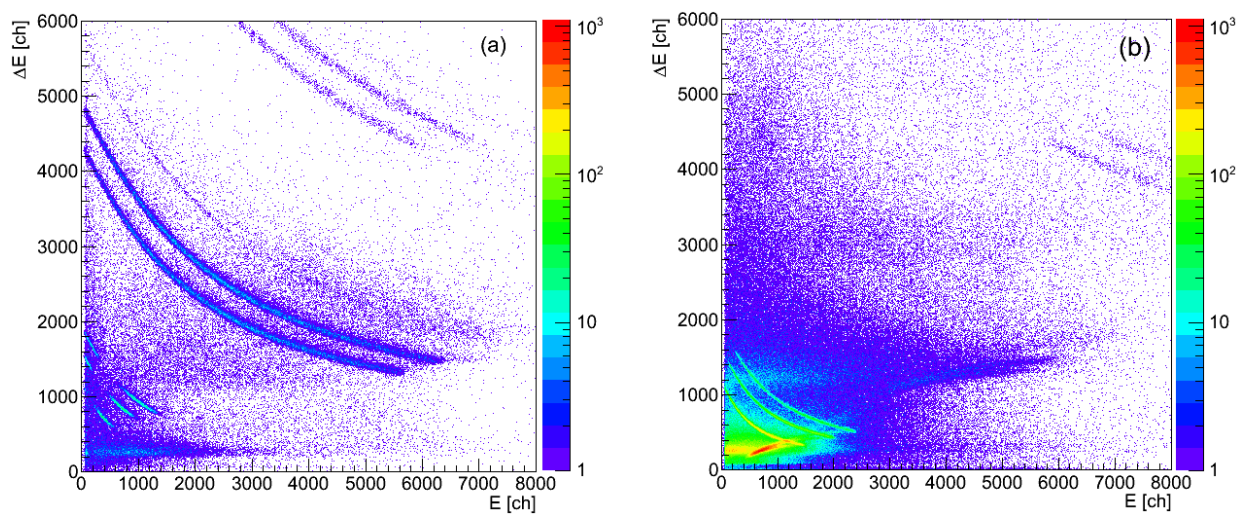

Fig. 2. $\Delta E-E$ maps obtained with the Artificial Neural Network selection criteria: (a) events recognized as a signal, (b) events classified as a background.

Plotting and analysing the real atomic mass distribution for the both neuron subsets, see Fig. 3, the background reduction efficiency has been estimated to be on the level of $75 \%$.

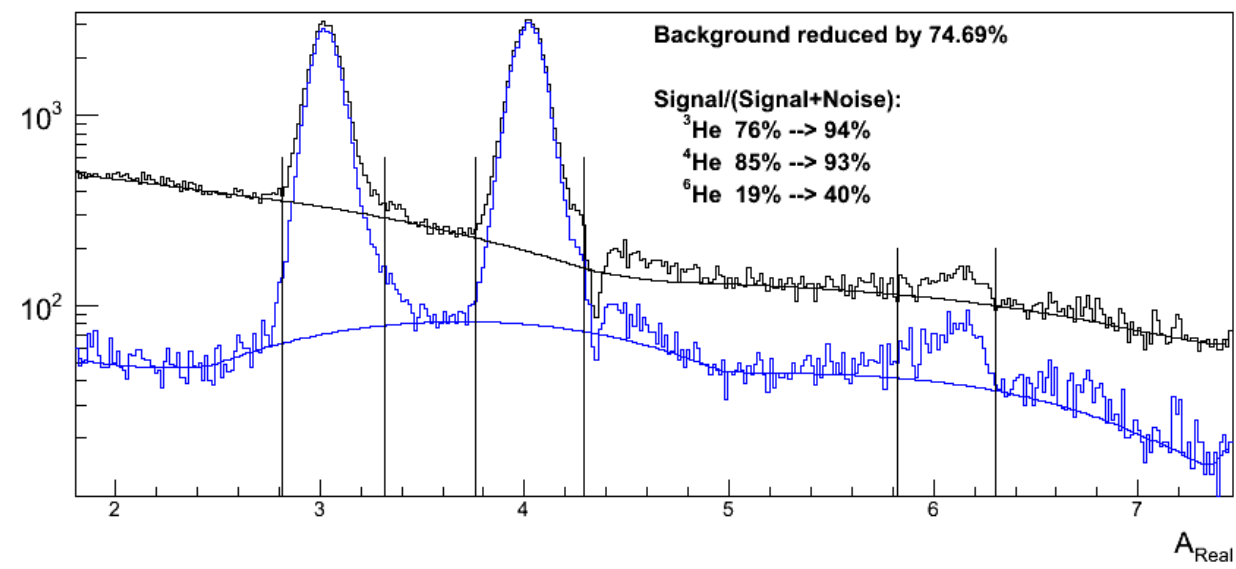

Fig. 3. The real mass number distribution along the calibration lines and the background reduction using KNN efficiency. Black line - original distribution, see Fig. 1 (a), gray/blue line - after background reduction, see Fig. 2 (a). 
The background recognition plays an important role in the KRATTA experimental data analysis. It allows to isolate the background hits underneath the isotopic lines and thus to perform reliable reconstruction of primary yields. The presented procedure was based on the Kohonen Neural Network and the analysis allowed to reduce the background by about $75 \%$.

We acknowledge the support by the Foundation for Polish Science MPD program, co-financed by the European Union within the European Regional Development Fund and funding by the Polish Ministry of Science and Higher Education under grant No. DPN/N108/GSI/2009.

\section{REFERENCES}

[1] J.B.A. England, Techniques in Nuclear Structure Physics, Wiley (1974).

[2] J. Łukasik et al., Nucl. Instrum. Methods Phys. Res. A 709, 120 (2013).

[3] P. Russotto et al., EPJ Web Conf. 31, 00012 (2012); IWM 2009 Conf. Proceedings, vol. 101, p. 22; Phys. Lett. B697, 471 (2011).

[4] J. Lukasik et al., Pulse Shape Analysis for KRATTA Modules in: ASY-EOS 2012 International Workshop on Nuclear Symmetry Energy and Reaction Mechanisms Proceedings.

[5] T. Kohonen, Biol. Cybern. 43, 59 (1982).

[6] ROOT website: root.cern.ch 